\title{
Atomic and Electronic Reconstruction at the a-LAO/STO Interface by E-Beam Induced Crystallization
}

Gwangyeob Lee ${ }^{1,2}$, Shin-ik Kim ${ }^{3}$, Gyeongtak Han ${ }^{4}$, Young-Min Kim ${ }^{4,5}$, Seung-Hyub Baek ${ }^{3}$, Do Hyang $\mathrm{Kim}^{2}$ and Hye Jung Chang ${ }^{1,6^{*}}$

1. Advanced Analysis Center, Korea Institute of Science and Technology, Seoul, South Korea.

2. Department of Materials Science and Engineering, Yonsei University, Seoul, South Korea.

3. Electronic Materials Research Center, Korea Institute of Science and Technology, Seoul, South Korea.

4. Department of Energy Science, Sungkyunkwan University, Suwon, South Korea.

5. IBS Center for Integrated Nanostructure Physics, Institute for Basic Science, Suwon, South Korea.

6. Division of Nano \& Information Technology, KIST School, University of Science and Technology, Seoul, South Korea.

* Corresponding author: almacore@kist.re.kr

Over the last several decades, researches on the perovskite oxides have been rapidly increasing with their promising applications [1]. In oxide materials, a strongly correlated electrons bring unique properties and the crystal structure of oxides is highly adaptable to changes at the interface. Thus, various intriguing physical properties are observed at the oxide interfaces [2].

A two-dimensional electron gas (2DEG) in $\mathrm{LaAlO}_{3} / \mathrm{SrTiO}_{3}(\mathrm{LAO} / \mathrm{STO})$ system is one of the most representative materials of the electron correlated novel properties. Many experimental as well as theoretical studies have been conducted to figure out the interfacial properties, and several major mechanisms have been suggested for the interfacial conductivity; polar catastrophe, oxygen vacancies and intermixing [3]. However, the origin of the interfacial conductivity still have not been fully understood, and any single scenario cannot account the various interfacial phenomena solely.

On the other hand, the 2DEG also can be formed at the amorphous(a)-LAO/STO interface, and it shows similar interfacial metallic behaviour to the 2DEG at the crystalline(c)-LAO/STO interface [4]. The mechanism for 2DEG formation at a-LAO/STO may also different from the c-LAO/STO since there is no polar discontinues at the a-LAO/STO interface. Therefore, many experimental as well as theoretical findings in c-LAO/STO system cannot be directly applied to a-LAO/STO heterostructure. Nevertheless, there are many advantages of the a-LAO/STO heterostructure in comparison with the c-LAO/STO such as simple film growth condition, various available compositions, and tuning of the transport properties by growth conditions [5]. In spite of these advantages, there are only few studies on the amorphous/crystalline oxide system so far while a lot of researches have been done on the crystalline/crystalline oxide heterostructures. Thus, further understanding on the fundamental mechanisms and interfacial phenomena at the amorphous/crystalline oxide heterostructures are necessary for the future oxide electronics.

Here, we directly investigated the change of the interfacial structure according to their physical properties. The a-LAO/STO heterostructure which shows unique interfacial property was used as a model system to demonstrate the strong correlation between interfacial property and atomic structure.

In this study, we observed the atomic as well as electronic reconstruction at the a-LAO/STO heterointerface during crystallization of the a-LAO overlayer. The accelerated electron beam (e-beam) in scanning transmission electron microscope (STEM) can epitaxially crystallize the various amorphous 
oxide materials [6]. This enables a real-time observation on the atomic/electronic structural changes simultaneously.

The observation of interatomic distance as well as octahedral distortions at the heterointerface were measured from the HAADF-STEM and ABF-STEM images respectively and the change of electronic structures were observed by electron energy loss spectroscopy (EELS). HAADF STEM images and electron energy loss spectra were collected by aberration-corrected STEM (Titan S80-300; FEI) at 300 $\mathrm{kV}$. The ABF STEM images were acquired using JEOL JEM-ARM300F.

The layer-by-layer epitaxial crystallization of a-LAO was performed by e-beam irradiation in STEM as shown in Figure 1(a). During the crystallization of the a-LAO from the interface, atomic/electronic structural changes near the interface were shown depending on the interfacial state. Also, the origin of the different atomic as well as electronic reconstructions such as the lattice expansion and Ti valence change near the heterointerface was discussed. This gives an account of how the interfacial structure correlated with the interfacial property. Using e-beam lithography, finally, conducting lines can be patterned into an insulating a-LAO matrix by growing the LAO above the critical thickness of 4 u.c. [7]. (Figure 1(b))

\section{References:}

[1] J Chakhalian, AJ Millis and J Rondinelli, Nature Materials 11(2) (2012), p. 92.

[2] HY Hwang et al., Nature Materials 11 (2012), p. 103.

[3] L Yu and A Zunger, Nature Communications 5 (2014), p. 5118.

[4] SY Moon et al.,Electronic Materials Letters 12(2) (2016), p. 243.

[5] CJ Li et al., Scientific Reports 8 (2018), p. 404.

[6] G Lee et al., RSC Advances 7(64) (2017), p. 40279.

[7] CW Schneider et al., Appl. Phys. Lett. 89 (2006), p. 122101.
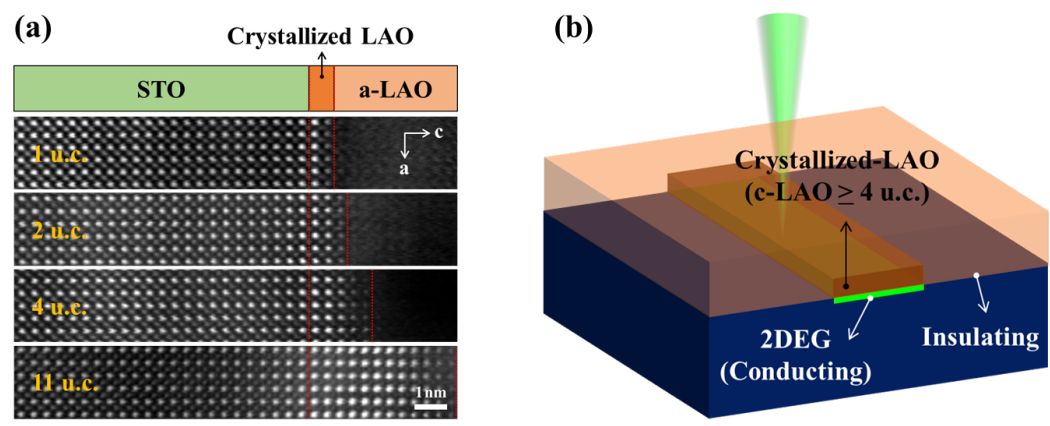

Figure 1. (a) The layer-by-layer crystallized LAO by e-beam irradiation in STEM. (b) A schematic describing e-beam induced 2 DEG patterning in insulating matrix. 